\title{
Thrombin conducts epithelial-mesenchymal transition via protease-activated receptor-1 in human gastric cancer
}

\author{
TADAYOSHI OTSUKI, DAISUKE FUJIMOTO, YASUO HIRONO, \\ TAKANORI GOI and AKIO YAMAGUCHI
}

\author{
First Department of Surgery, Faculty of Medicine, University of Fukui, Fukui 910-1193, Japan
}

Received July 8, 2014; Accepted August 21, 2014

DOI: 10.3892/ijo.2014.2651

\begin{abstract}
Epithelial-mesenchymal transition (EMT) is thought to be a key step for cancer metastasis. Using an immunohistochemical approach with gastric carcinoma tissue, we found the expression of protease-activated receptor-1 (PAR1), along with a metalloproteinase known to activate PAR1, were associated with poorer prognosis, compared with expression-negative tumors, and activated PAR1 promotes gastric cancer cell invasion and proliferation in vivo. In this study we observed EMT induction by the PAR1 agonist $\alpha$-thrombin, in human gastric cell lines stably expressing PAR1. We investigated $\alpha$-thrombin-induced changes in the cell forms of pcDNA3.1-MKN45 (MKN45/Mock), pcDNA3.1-PAR1 transfected MKN45 (MKN45/PAR1), and MKN74. Expression levels of epithelial and mesenchymal markers as well as the distribution of transcriptional factors of E-cadherin in the cytoplasm and nucleus were also noted in these cell lines. We observed $\alpha$-thrombin-induced morphological changes in MKN45/PAR1 and MKN74 cells. Western blotting and immunohistochemistry of these cells indicated a fall in the expression level of E-cadherin and an increase in fibronectin expression after $48 \mathrm{~h}$. PAR1 activation also induced significant increases in nuclear levels of the Snail which is a repressor of E-cadherin gene expression. We found EMT in gastric cancer cell lines that underwent $\alpha$-thrombininduced PAR1 activation.
\end{abstract}

\section{Introduction}

Gastric cancer is one of the most fatal cancers, especially in eastern Asia. In recent years, despite the progress made in diagnosis and treatment of gastric cancer, the mortality rate of gastric cancer remains high, mainly due to complex biological characteristics of gastric cancer and its high rate of

Correspondence to: Dr Daisuke Fujimoto, First Department of Surgery, Faculty of Medicine, University of Fukui, 23-3 Matsuoka Shimoaiduki, Eiheiji-cho, Yoshida-gun, Fukui 910-1193, Japan E-mail: fujimoto@u-fukui.ac.jp

Key words: protease-activated receptor-1, gastric cancer, epithelialmesenchymal transition malignancy (1). Most patients are diagnosed at an advanced stage and therefore it remains one of the most life-threatening pathological events involving the spread of tumor cells from a primary tumor to other organs. Currently, some studies have described the metastasis of gastric cancer, but its exact mechanism remains unknown. Similar to tumorigenesis, tumor metastasis also includes multiple factors and genes. Metastasis consists of five distinct steps: i) detachment of tumor cells from the primary tumor; ii) invasion into surrounding tissue; iii) intravasation into blood or lymphatic vessels; iv) dissemination in the blood stream or the lymphatic system; and, v) extravasation and outgrowth at secondary sites. To detach from the primary tumor and to invade into the surrounding tissue, tumor cells need to dismantle cell-cell junction, remodel cell-matrix contact sites, and follow a chemo-attractive path through the extracellular matrix, mined by secreted proteinases. These processes are commonly observed in various normal conditions, such as in developmental processes i.e. gastrulation or neural crest cell migration, where differentiated, epithelial cells dedifferentiate, move to a distant site, and then re-differentiate to form a new structure. This temporary and reversible phenomenon is known as the epithelial-mesenchymal transition (EMT), a process that is currently in the limelight of investigating the onset of cancer cell migration, invasion and metastatic dissemination $(2,3)$. Recent studies have shown that abnormal EMT is closely related to tumorigenesis and progression of epithelial cancers, such as lung cancer, colon cancer and breast cancer $(4,5)$.

The association of thrombin and cancer is documented (6). But, the role of thrombin in propagation of the pro-malignant phenotype has not been fully elucidated. This pro-malignant role of thrombin has been attributed to its stimulation of tumor adhesion (7-10), growth (11), metastasis (12-14), and angiogenesis $(15,16)$. Thrombin, a multifunctional serine protease, activates platelets and regulates the behavior of other cells through G-protein coupled protease-activated receptors (PARs) (17,18). Protease-activated receptor-1 (PAR1), the prototype of this family, is activated when thrombin cleaves the amino-terminal extracellular domain at a specific site (19). PAR1 is overexpressed in invasive and metastatic tumors and the expression levels directly correlate with the degree of invasiveness of the cancer. PAR1 has been found to be instrumental in cell growth and invasion of tumor-derived cells $(20,21)$. 
We previously reported that the expression of PAR1, along with a metalloproteinase known to activate PAR1 (MMP1) were associated with poorer prognoses, compared with expression-negative tumors, and activated PAR1 promotes gastric cancer cell invasion in vivo as well as cell morphological changes (22-24). These results underline the relevance of EMT and PAR1 activity to gastric cancer, however there are few reports on the role of PAR1 in EMT. Therefore, this study investigated the role of PAR1 activity in EMT by observing the expression levels of epithelial and mesenchymal markers, such as E-cadherin and vimentin. This study on the relationship between PAR1 activity and EMT in gastric cancer further elucidates metastasis which in turn could serve to impact gastric cancer prognoses and potentially add knowledge for targeted therapy.

\section{Materials and methods}

Reagents. An antibody against PAR1 (clone WEDE15) was purchased from Beckman Coulter (Fullerton, CA, USA). Anti-E-cadherin, fibronectin, vimentin, $\beta$-catenin, Twist, E12/E47 and Snail were purchased from Abcam (Cambridge, UK). Anti-GAPDH was from Imgenex (San Diego, CA, USA). Human $\alpha$-thrombin was purchased from Sigma-Aldrich (catalog no. T1063) (St. Louis, MO, USA). The selective PAR1 antagonist SCH79797 (catalog no. 1592) (IC50=70 nM) was purchased from Tocris Bioscience (Avonmouth, UK) (25).

Cell culture. The human gastric cancer cell lines, MKN45 and MKN74 cells, were obtained from the Riken Cell Bank (Tsukuba, Japan). Cells were cultured at $37^{\circ} \mathrm{C}$ in $5 \% \mathrm{CO}_{2}$ in RPMI-1640 medium containing $10 \%$ fetal bovine serum (FBS). Cells were propagated by mechanical re-suspension using a scraper, without the use of trypsin. PAR1 is expressed in MKN74, not in MKN45 (23).

Western blot assay. Whole, cytoplasmic and nuclear cell protein was extracted using RIPA-buffer (Wako, Osaka, Japan) containing $1 \%$ protease inhibitor cocktail (Sigma-Aldrich) and NE-PER (Thermo Scientific, Rockford, IL, USA). Protein concentration was measured with a protein assay reagent (Bio-Rad, Hercules, CA, USA). Proteins were resolved by SDS-PAGE using a 5-20\% SuperSep gel (Wako) and analyzed by western blotting using polyvinylidene difluoride membranes (Millipore, Bedford, MA, USA) according to the manufacturer's instructions. Membranes were blocked with $3 \%$ non-fat dry milk in TBS plus $0.1 \%$ Tween-20 (TBST). The membranes were probed with anti-E-cadherin antibody at 1:1,000 dilution, anti-fibronectin and anti-vimentin antibody at 1:750 dilution, anti- $\beta$-catenin antibody at 1:2,000 in TBST. Protein bands were incubated with primary antibody overnight at $4^{\circ} \mathrm{C}$. An enhanced chemiluminescence detection system (ImmunoStar; Wako) was used for visualized of immunoreactive bands after the reaction with the HRP-labeled secondary antibody against mouse or rabbit IgG. Equal loading of samples was confirmed by probing the membranes with GAPDH antibody.

Measurement of Snail activation by electrophoretic mobility shift assay. MKN45/mock, MKN45/PAR1 and MKN74 were treated for $24 \mathrm{~h}$ with $15 \mathrm{nM} \alpha$-thrombin or $15 \mathrm{nM} \alpha$-thrombin and $70 \mathrm{nM} \mathrm{SCH79797.} \mathrm{Nuclear} \mathrm{fractions} \mathrm{were} \mathrm{extracted} \mathrm{from}$ the cultured cells using NE-PER (Thermo Scientific).

In the electrophoretic mobility shift assay (EMSA), the double-stranded oligonucleotide probes were labeled with biotin. Non-labeled oligonucleotides were used as competitors for biotin-labeled oligonucleotide binding. The sequences flanking the E-boxes are for E-box 1, 5'-CTGAGATTAC ATGCGTGAGTCACTA-3'; for E-box 2, 5'-TGTTGTGTG CATGTGTTATGGAACC-3'; and for E-box 3, 5'-ATTACA ACACGAGCCCACCATGCCT-3'. Briefly, $20 \mu \mathrm{l}$ of binding reaction containing $2 \mu \mathrm{g}$ of nuclear extract was prepared according to the kit instruction manual and incubated at room temperature for $20 \mathrm{~min}$. Complexes were resolved on $5-20 \%$ SuperSep gel (Wako) in $0.5 \mathrm{X}$ TBE buffer at $4^{\circ} \mathrm{C}$. When electrophoresis was completed, binding reactions were electrophoretically transferred to $\mathrm{N}+$ nylon membrane. The transferred DNA then was cross-linked to the $\mathrm{N}+$ nylon membrane, and the biotin-labeled DNA-protein complex was detected by streptavidin-HRP using a LightShift Chemiluminescent EMSA kit (Thermo Scientific).

Immunofluorescence. Cultured cells were fixed with $4 \%$ paraformaldehyde at room temperature, permeabilized with $0.1 \%$ Triton $\mathrm{X}-100$ in PBS and blocked with $3 \%$ FBS in PBS. Following overnight incubation at $4^{\circ} \mathrm{C}$ with primary antibodies, and incubation in the dark with Alexa 405 and 488 Fluor dye-labeled secondary antibodies, immunofluorescence was detected using a Leica DMLB confocal laser fluorescence microscope (Leica Microsystems, Mannheim, Germany).

\section{Results}

PARl activation via $\alpha$-thrombin induces epithelial marker downregulation and mesenchymal marker upregulation. Given the shape change caused by $\alpha$-thrombin activation of PAR1 (24), we hypothesized that EMT would have been induced in gastric cancer cells. To test this hypothesis, we collected cell lysate from MKN45/mock, MKN45/PAR1 and MKN74 treated with $15 \mathrm{nM} \alpha$-thrombin or $15 \mathrm{nM}$ $\alpha$-thrombin and $70 \mathrm{nM}$ SCH79797. When MKN45/mock was treated with $\alpha$-thrombin or $\alpha$-thrombin plus SCH7979 for $24 \mathrm{~h}$, we showed that the epithelial and mesenchymal markers were stably expressed at a level that did not change over time (Fig. 1A). MKN45/PAR1 and MKN74 treated with $\alpha$-thrombin presented reduced expression levels of the epithelial markers E-cadherin and $\beta$-catenin in cytoplasmic lysate and an icreased expression level of $\beta$-catenin in nuclear lysate. On the other hand, the expression levels of fibronectin and vimentin, which are mesenchymal markers, were increased in cytoplasmic lysate (Fig. 1B). MKN45/PAR1 and MKN74 treated with $\alpha$-thrombin along with $\mathrm{SCH} 79797$ presented expression levels of fibronectin and vimentin similar to that of MKN45/mock (Fig. 1C).

Immunofluorescence of E-cadherin and fibronectin expression. Our investigation of the role of thrombin in the morphology of MKN45/PAR1 and MKN74 cells employed immunofluorescence staining to visualize the expression levels of E-cadherin and fibronectin in untreated MKN45/mock, MKN45/PAR1 
A

epithelial marker mesenchymal marker

MKN45/mock

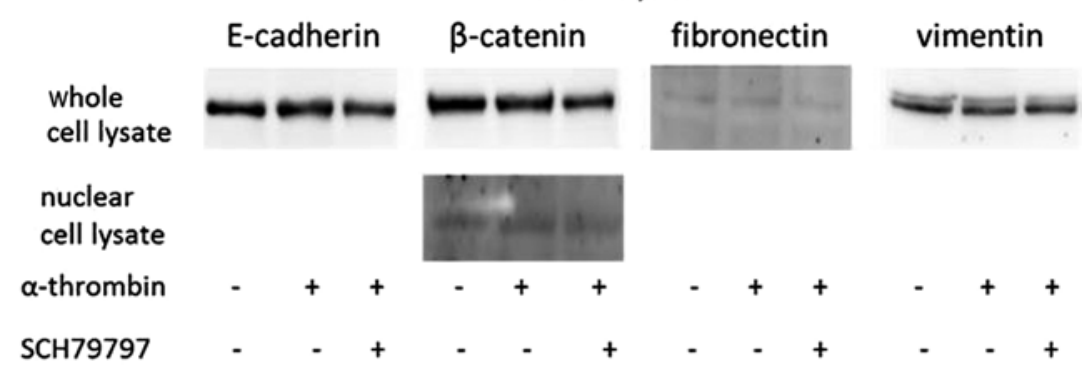

B

epithelial marker mesenchymal marker
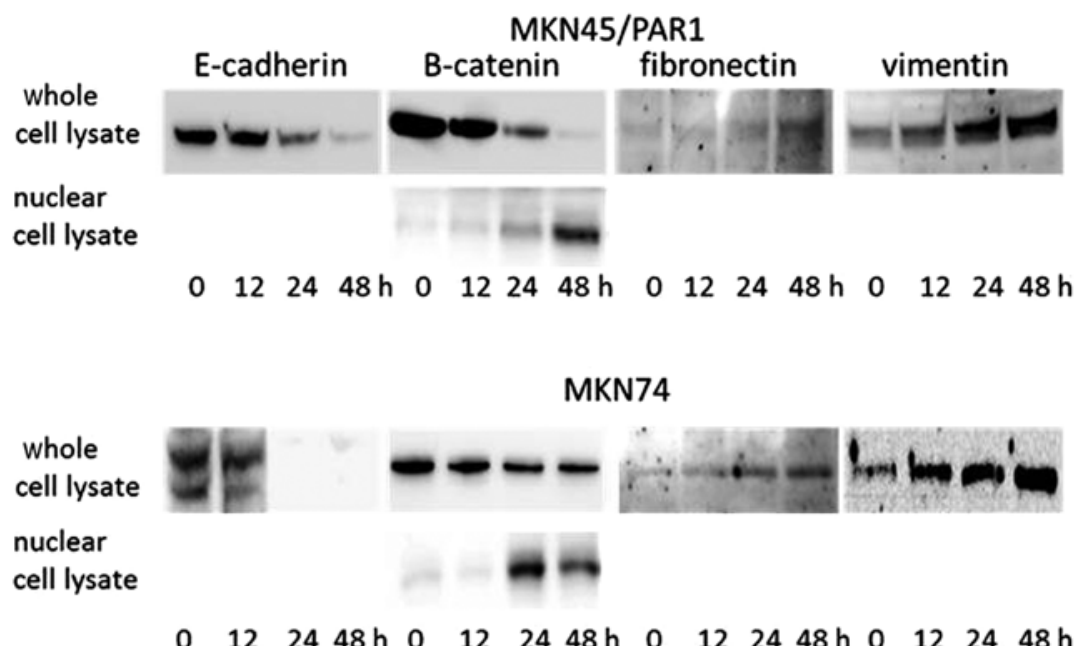

Exposure time by $\alpha$-thrombin

C

epithelial marker

mesenchymal marker

MKN45/PAR1
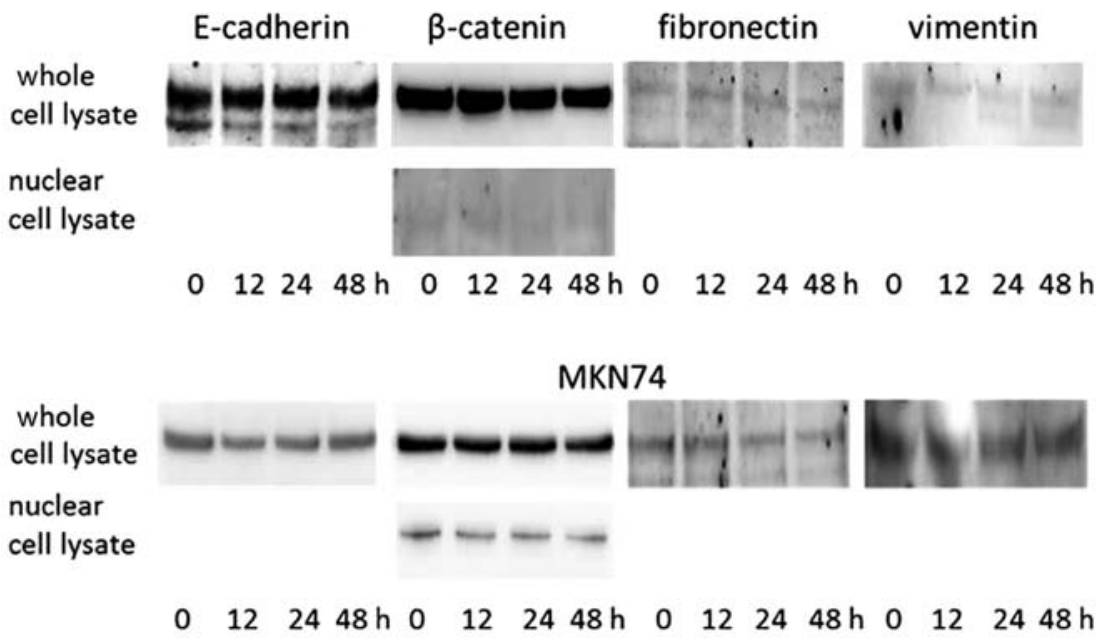

Exposure time by $\alpha$-thrombin and $\mathrm{SCH} 79797$

Figure 1. Protease-activated receptor-1 (PAR1) activation-dependent expression of epithelial-mesenchymal transition (EMT) markers in MKN45/PAR1 and MKN74 cells. Whole-cell and nuclear-cell lysates of MKN45/mock, MKN45/PAR1 and MKN74 cells were probed for EMT-marker expression by western blotting. (A) EMT-marker expression remains the same in MKN45/mock cells treated with $\alpha$-thrombin or $\alpha$-thrombin plus SCH79797 for 24 h. (B) MKN45/PAR1 and MKN74 cells treated with $\alpha$-thrombin present decreased levels of E-cadherin and $\beta$-catenin and increased levels of fibronectin and vimentin in whole-cell lysates over time. Nuclear lysates of these $\alpha$-thrombin-treated cells presented an increase in $\beta$-catenin over time suggesting movement of $\beta$-catenin into the nucleus. (C) When treated with $\alpha$-thrombin and SCH79797, MKN45/PAR1 and MKN74 cells present results similar to MKN45/mock cells. Equal loading of protein was confirmed with anti-GAPDH antibody. 
A

\section{$\alpha$-thrombin \\ SCH79797}

fibronectin

E-cadherin

B

\section{$\alpha$-thrombin \\ SCH79797}

fibronectin

E-cadherin
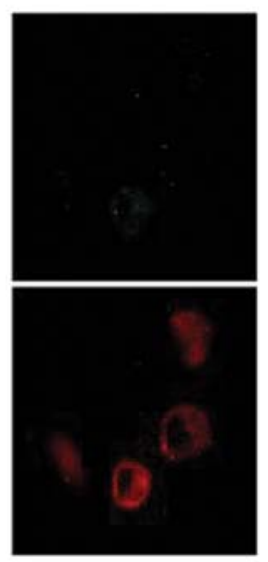

C

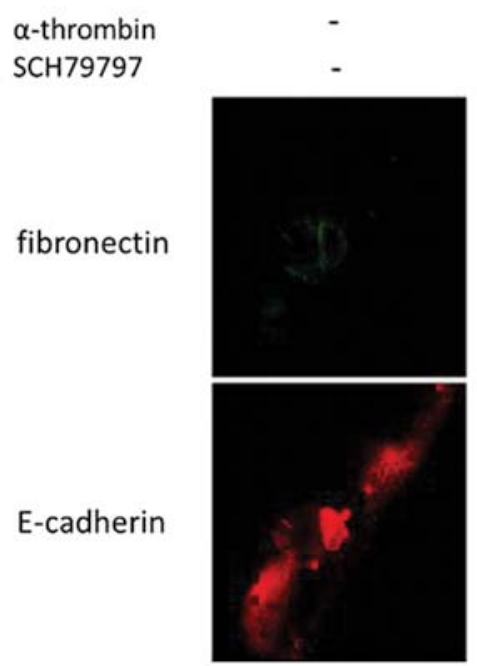

MKN45/mock
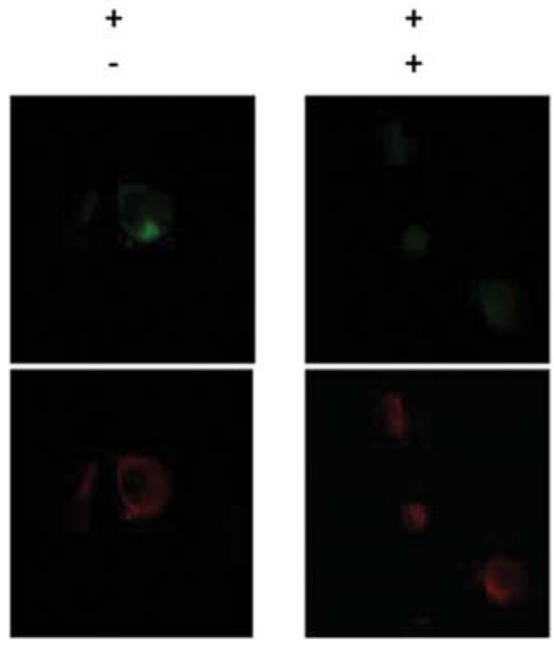

\section{MKN45/PAR1}
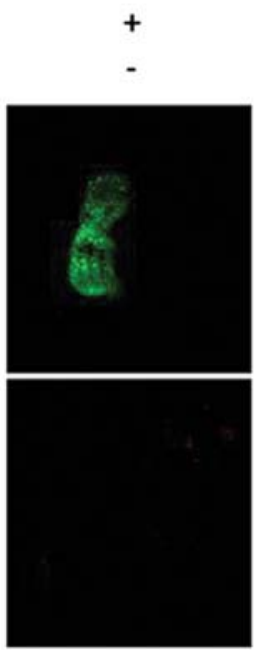

MKN74
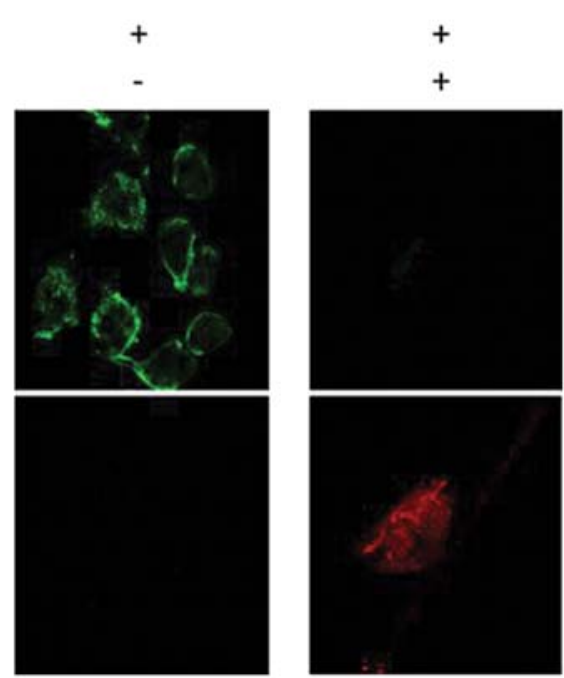

Figure 2. Fluorescence immunocytochemical staining of fibronectin and E-cadherin in MKN45/mock, MKN45/PAR1 and MKN74 cells, when treated with $\alpha$-thrombin or $\alpha$-thrombin plus SCH79797. (A) MKN45/mock cells present no significant changes in fibronectin and E-cadherin expression. (B) MKN45/APR1 and MKN74 cells treated with $\alpha$-thrombin presented a decreased level of E-cadherin expression and an enhanced level of fibronectin expression. (C) MKN45/APR1 and MKN74 cells treated with $\alpha$-thrombin and SCH79797, presented fibronectin and E-cadherin expression levels similar to that of untreated cultures of these cells. 

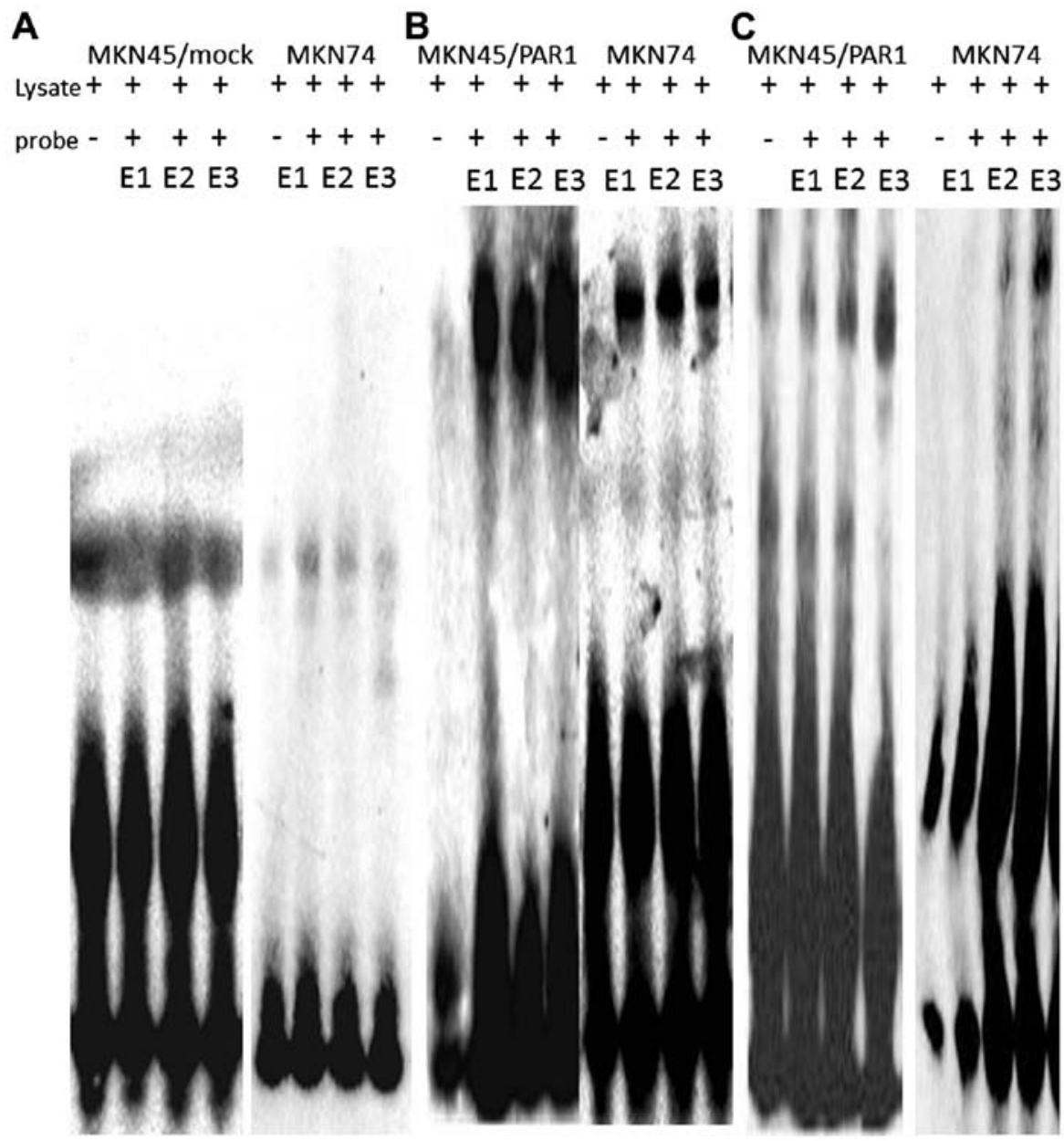

Figure 3. Electrophoretic mobility shift assays (EMSAs), demonstrate specific interaction with the E-cadherin promoter. (A) EMSAs were performed with nuclear extracts from MKN45/mock and MKN74 cells and demonstrate an E-cadherin E-box 1-3 nuclear protein complex. No levels of specific E-box complexes are seen in MKN45/mock and MKN74 cells. These cells are the control. (B) These lanes show nuclear extracts from MKN45/PAR1 and MKN74 cells, treated with $\alpha$-thrombin for $12 \mathrm{~h}$, and demonstrate an E-cadherin E-box1-3 nuclear protein complex. Higher levels of specific E-box complexes are seen in MKN45/PAR1 and MKN74 cells treated with $\alpha$-thrombin that overexpress relative to control cells. (C) Specific complexes were inhibited by protease-activated receptor-1 (PAR1) selective antagonist SCH79797.

and MKN74 cells and these cells were treated with $15 \mathrm{nM}$ $\alpha$-thrombin in the absence and presence of $70 \mathrm{nM} \mathrm{SCH79797.}$

MKN45/PAR1 and MKN74 cells underwent a morphological change from round- or epithelial-like to a spindle-like shape phenotype upon treatment with $\alpha$-thrombin and this morphological change was not observed in response to treatment with a combination of $\alpha$-thrombin and SCH79797 (Fig. 2). The expression of E-cadherin decreased and the expression of fibronectin increased in response to treatment of MKN45/PAR1 and MKN74 cells with $\alpha$-thrombin while no such change in expression levels were observed in these cells that were either untreated or underwent $\alpha$-thrombin treatment in the presence of SCH79797 (Fig. 2).

Snail, transcriptional factor, travels into the nucleus. Next, we investigated the impact of $\alpha$-thrombin induced PAR1 activation upon E-cadherin expression. Several E-cadherin transcriptional repressors have been characterized (Snail, E12/E47, Twist, and SIP-1) and shown to interact with proximal E-boxes of the E-cadherin promoter.

To test whether or not PAR1 agonist-mediated elevation is associated with an increase in binding to the E-cadherin promoter, we examined the binding of these transcriptional repressors to E-boxes by means of EMSA and western blotting. Assessment was carried out of E-cadherin promoter E-box binding by nuclear proteins from MKN45/mock and MKN74 as control, MKN45/PAR1- and MKN74-treated $\alpha$-thrombin, and MKN45/PAR1 and MKN74 treated with $\alpha$-thrombin and SCH79797. We designed oligonucleotide probes comprising E-box binding sequences as described previously (26). No shifted band was evident when the labeled oligonucleotide was exposed to nuclear lysates derived from MKN45/mock and MKN74 cells (Fig. 3A). In contrast, labeled oligonucleotide incubated with nuclear lysate from MKN45/PAR1 and MKN74 cells with $\alpha$-thrombin clearly shows a retarded electrophoretic mobility band (Fig. 3B). Slightly leaky expression of a shifted band was observed when the labelled oligonucleotide was incubated with nuclear lysate from MKN45/PAR1 and MKN74 cells treated with a combination of $\alpha$-thrombin and SCH79797 (Fig. 3C).

Subsequently, we employed western blotting to examine nuclear expression of Twist, Snail and E12/E47. Twist and E12/E47 were not detected in nuclear lysates of MKN45/PAR1 and MKN74 that underwent $\alpha$-thrombin induced PAR1 activation (Fig. 4). Snail was present in the nuclear lysates derived 
MKN45/PAR1

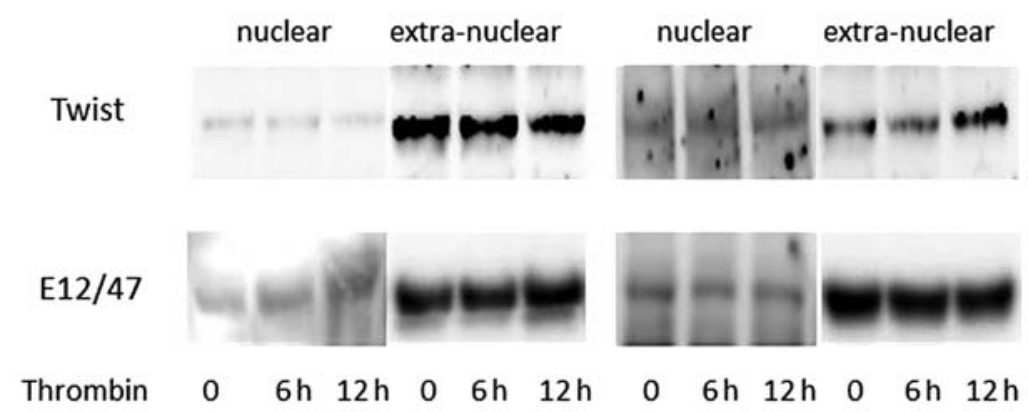

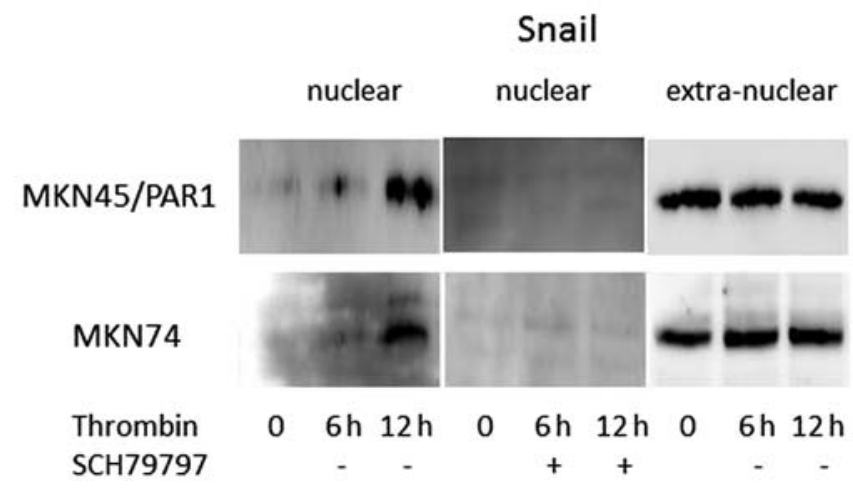

Figure 4. Snail detected in nuclear lysate. The impact of $\alpha$-thrombin treatment of MKN45/PAR1 and MKN74 cells upon nuclear localization of transcription factors in these cells (Twist, Snail and E12/E47) was profiled by means of western blotting. Twist and E12/E47 were not able to migrate into the nucleus, when treated with $\alpha$-thrombin while Snail was able to do so, when these cells were treated with $\alpha$-thrombin for $12 \mathrm{~h}$.

from MKN45/PAR1 and MKN74 that underwent $\alpha$-thrombin induced PAR1 activation while Snail was not detected in nuclear lysates of these cells in which $\alpha$-thrombin-induced PAR1 activation was suppressed by SCH79797 (Fig. 4).

\section{Discussion}

In the current study, we demonstrate that PAR1 activity induces pro-migratory effects in gastric cancer cell lines by inducing EMT. Malignant tumors including gastric cancer are multi-step processes associated with changes in gene expression involved in cell proliferation, invasion, and metastasis. The most troubling metastasis in gastric cancer is peritoneal dissemination, for which anticancer therapies have been developed. However, despite the proven benefits of these treatments $(27,28)$, patients with peritoneal dissemination of gastric cancer still have poor prognosis and outcomes of gastric cancer require further improvement.

Many metastasis-related proteins have been reported in various cancers including gastric cancer. PAR1, G-protein coupled receptor, was identified as one of the most upregulated proteins in advanced gastric cancer by immunohistochemistory (23). PAR1, also known as the coagulation factor II (thrombin) receptor, is a protein that in humans is encoding the F2R gene (29). Thrombin activates PAR1 in alveolar epithelial cells and this pathway is associated with epithelial to myofibroblats transition and collagen secretion, and thrombin-induced EMT is mediated through PAR1 (30). Additionally, PAR1-mediated extracellular signal-regulated kinase activation and promoted the transition of tubular epithelial cells into cells of mesenchymal phenotype.

Recent studies have revealed that PAR1 activity encouraged cancer invasion in various cancers $(23,31,32)$. EMT, characterized by a gain of mesenchymal cell markers (fibronectin, vimentin, smooth muscle actin, and $\mathrm{N}$-cadherin) and a loss of epithelial markers (E-cadherin and $\beta$-catenins) $(33,34)$, is a process whereby cells undergo molecular alterations that facilitate cell motility and invasion (35). In this study we shown that thrombin via PAR1 induced EMT in gastric cancer MKN45/PAR1 and MKN74 cell lines and this was associated with time-dependent downregulation of E-cadherin accompanied by an upregulation of vimentin and $\beta$-catenin. Thrombin also induced a morphological alteration in MKN45/PAR1 and MKN74 cells to a spindle-shaped phenotype. Additionally, we show that expression of E-cadherin, vimentin and $\beta$-catenin remained unchanged in MKN45/PAR1 cells exposed to SCH79797, which is a specific PAR1 antagonist. The most common biochemical change associated with EMT is the loss of E-cadherin expression. These data imply that PAR1 activation by thrombin induces EMT in gastric cancer and may be conducive to invasion and metastasis.

E-cadherin transcriptional repressors such as Snail, Slug, E12/E47, and Twist have traditionally been implicated in promoting EMT in various systems of embryonic development and tumor progression $(36,37)$. The transcription factor Twist is a component of embryonic morphogenesis (38). Snail, a Zinc-finger transcription factor, has been isolated in Drosophila embryos (39). During the embryonic development, 
Snail has been implicated in the triggering of EMT in the precursor cells of the mesoderm and neural crest (40). Snail is known to directly repress E-cadherin gene transcription by binding to the E-box on the E-cadherin promoter (41). Several reports have also implicated that Snail not only in E-cadherin repression but also in the acceleration of cancer invasion in various carcinomas $(41,42)$. Snail expression correlated with histological grade and lymph node status in breast carcinomas (43), and Snail mRNA expression levels independently correlated with capsular invasion in hepatocellular carcinoma (44). We showed by means of western blotting and EMSA that the transcription factor Snail moved from cell cytoplasm to the nucleus. These data indicated that PAR1 activation via thrombin induced Snail-mediated E-cadherin transcriptional repressor and ultimately EMT in gastric cancer cells.

We conclude that at least one of the facets of PAR1 activity by thrombin induced upregulation of malignant phenotype is via the upregulation of Snail in gastric cancer cells. SCH79797, which is a PAR1 antagonist, blocked these effects of thrombin. Thrombin, MMP1 and urokinase-type plasminogen activator, which are PAR1 agonist, contribute to tumor invasion and tumorigenesis $(45,46)$. These results suggest that inhibition of PAR1 signaling pathway may be a new strategy to prevent tumor invasion and metastasis.

\section{References}

1. Memon MA, Subramanya MS, Khan S, Hossain MB, Osland E and Memon B: Meta-analysis of D1 versus D2 gastrectomy for gastric adenocarcinoma. Ann Surg 253: 900-911, 2011.

2. Thiery JP and Sleeman JP: Complex networks orchestrate epithelial-mesenchymal transitions. Nat Rev Mol Cell Biol 7: 131-142, 2006.

3. Grünert S, Jechlinger $\mathrm{M}$ and Beug H: Diverse cellular and molecular mechanisms contribute to epithelial plasticity and metastasis. Nat Rev Mol Cell Biol 4: 657-665, 2003.

4. Jain VK and Cunningham D: Targeting angiogenesis in advanced gastric cancer: Is this end of the road? Transl Gastrointest Cancer 1: 119-121, 2012.

5. Rose CS and Malcolm S: A TWIST in development. Trends Genet 13: 384-387, 1997.

6. Nierodzik ML and Karpatkin S: Thrombin induces tumor growth, metastasis, and angiogenesis: evidence for a thrombin-regulated dormant tumor phenotype. Cancer Cell 10: 355-362, 2006.

7. Nierodzik ML, Plotkin A, Kajumo F and Karpatkin S: Thrombin stimulates tumor-platelet adhesion in vitro and metastasis in vivo. J Clin Invest 87: 229-236, 1991.

8. Nierodzik ML, Kajumo F and Karpatkin S: Effect of thrombin treatment of tumor cells on adhesion of tumor cells to platelets in vitro and tumor metastasis in vivo. Cancer Res 52: 3267-3272, 1992.

9. Nierodzik ML, Bain RM, Liu LX, Shivji M, Takeshita K and Karpatkin S: Presence of the seven transmembrane thrombin receptor on human tumour cells: effect of activation on tumour adhesion to platelets and tumor tyrosine phosphorylation. Br J Haematol 92: 452-457, 1996.

10. Klepfish A, Greco MA and Karpatkin S: Thrombin stimulates melanoma tumor-cell binding to endothelial cells and subendothelial matrix. Int J Cancer 53: 978-982, 1993.

11. Zain J, Huang YQ, Feng X, Nierodzik ML, Li JJ and Karpatkin S: Concentration dependent dual effect of thrombin on impaired growth/apoptosis or mitogenesis in tumor cells. Blood 95 3133-3138, 2000

12. Nierodzik ML, Chen K, Takeshita K, et al: Protease-activated receptor 1 (PAR-1) is required and rate-limiting for thrombin-enhanced experimental pulmonary metastasis. Blood 92: 3694-3700, 1998.

13. Hu L, Lee M, Campbell W, Perez-Soler R and Karpatkin S: Role of endogenous thrombin in tumor implantation, seeding and spontaneous metastasis. Blood 104: 2746-2751, 2004
14. Wojtukiewicz MZ, Tang DG, Ciarelli JJ, et al: Thrombin increases the metastatic potential of tumor cells. Int J Cancer 54: 793-806, 1993.

15. Huang YQ, Li JJ, Hu L, Lee M and Karpatkin S: Thrombin induces increased expression and secretion of VEGF from human FS4 fibroblasts, DU145 prostate cells and CHRF megakaryocytes. Thromb Haemost 86: 1094-1098, 2001.

16. Caunt M, Huang YQ, Brooks PC and Karpatkin S: Thrombin induces neoangiogenesis in the chick chorioallantoic membrane. J Thromb Haemost 1: 2097-2102, 2003.

17. Coughlin SR: How the protease thrombin talks to cells. Proc Natl Acad Sci USA 96: 11023-11027, 1999.

18. Vu TK, Hung DT, Wheaton VI and Coughlin SR: Molecular cloning of a functional thrombin receptor reveals a novel proteolytic mechanism of receptor activation. Cell 64: 1057-1068, 1991.

19. Rasmussen UB, Vouret-Craviari V, Jallat S, et al: cDNA cloning and expression of a hamster alpha-thrombin receptor coupled to $\mathrm{Ca}^{2+}$ mobilization. FEBS Lett 288: 123-128, 1991.

20. Even-Ram S, Uziely B, Cohen P, et al: Thrombin receptor overexpression in malignant and physiological invasion processes. Nat Med 4: 909-914, 1998.

21. Even-Ram SC, Maoz M, Pokroy E, et al: Tumor cell invasion is promoted by activation of protease activated receptor-1 in cooperation with the alpha vbeta 5 integrin. J Biol Chem 276: 10952-10962, 2001.

22. Fujimoto D, Hirono Y, Goi T, Katayama K and Yamaguchi A: Prognostic value of protease-activated receptor-1 (PAR-1) and matrix metalloproteinase-1 (MMP-1) in gastric cancer. Anticancer Res 28: 847-854, 2008.

23. Fujimoto D, Hirono Y, Goi T, Katayama K, Matsukawa S and Yamaguchi A: The activation of proteinase-activated receptor-1 (PAR1) mediates gastric cancer cell proliferation and invasion. BMC Cancer 10: 443, 2010.

24. Fujimoto D, Hirono Y, Goi T, Katayama K, Matsukawa S and Yamaguchi A: The activation of proteinase-activated receptor-1 (PAR1) promotes gastric cancer cell alteration of cellular morphology related to cell motility and invasion. Int J Oncol 42: 565-573, 2013.

25. Ahn HS, Foster C, Boykow G, Stamford A, Manna M and Graziano M: Inhibition of cellular action of thrombin by N3-cyclopropyl-7-[[4-(1-methylethyl)phenyl]methyl]7H-pyrrolo[3, 2-f]quinazoline-1,3-diamine (SCH 79797), a nonpeptide thrombin receptor antagonist. Biochem Pharmacol 60: 1425-1434, 2000.

26. Manohar CF, Bray JA, Salwen HR, et al: MYCN-mediated regulation of the MRP1 promoter in human neuroblastoma. Oncogene 23: 753-762, 2004.

27. Fushida S, Kinoshita J, Yagi Y, et al: Dual anti-cancer effects of weekly intraperitoneal docetaxel in treatment of advanced gastric cancer patients with peritoneal carcinomatosis: A feasibility and pharmacokinetic study. Oncol Rep 19: 1305-1310, 2008.

28. Yonemura Y, Elnemr A, Endou Y, et al: Multidisciplinary therapy for treatment of patients with peritoneal carcinomatosis from gastric cancer. World J Gastrointest Oncol 2: 85-97, 2010.

29. Coughlin SR, Vu TK, Hung DT and Wheaton VI: Characterization of a functional thrombin receptor. Issues and opportunities. J Clin Invest 89: 351-355, 1992.

30. Song JS, Kang CM, Park CK and Yoon HK: Thrombin induces epithelial-mesenchymal transition via PAR-1, PKC, and ERK1/2 pathways in A549 cells. Exp Lung Res 39: 336-348, 2013.

31. Juncker-Jensen A, Deryugina EI, Rimann I, et al: Tumor MMP-1 activates endothelial PAR1 to facilitate vascular intravasation and metastatic dissemination. Cancer Res 73: 4196-4211, 2013.

32. Ohshiro K, Bui-Nguyen TM, Divijendra Natha RS, Schwartz AM, Levine P and Kumar R: Thrombin stimulation of inflammatory breast cancer cells leads to aggressiveness via the EGFR-PAR1-Pak1 pathway. Int J Biol Markers 27: e305-e313, 2012.

33. Radisky DC: Epithelial-mesenchymal transition. J Cell Sci 118: 4325-4326, 2005.

34. Thiery JP: Epithelial-mesenchymal transitions in development and pathologies. Curr Opin Cell Biol 15: 740-746, 2003.

35. Yang J, Mani SA and Weinberg RA: Exploring a new twist on tumor metastasis. Cancer Res 66: 4549-4552, 2006.

36. Peinado H, Portillo F and Cano A: Transcriptional regulation of cadherins during development and carcinogenesis. Int J Dev Biol 48: 365-375, 2004. 
37. Yang J, Mani SA, Donaher JL, et al: Twist, a master regulator of morphogenesis, plays an essential role in tumor metastasis. Cell 117: 927-939, 2004.

38. Thisse B, el Messal M and Perrin-Schmitt F: The twist gene: isolation of a Drosophila zygotic gene necessary for the establishment of dorsoventral pattern. Nucleic Acids Res 15: 3439-3453, 1987.

39. Grau Y, Carteret C and Simpson P: Mutations and chromosomal rearrangements affecting the expression of snail, a gene involved in embryonic patterning in Drosophila melanogaster. Genetics 108: 347-360, 1984

40. Cano A, Pérez-Moreno MA, Rodrigo I, et al: The transcription factor snail controls epithelial-mesenchymal transitions by repressing E-cadherin expression. Nat Cell Biol 2: 76-83, 2000.

41. Batlle E, Sancho E, Franci C, et al: The transcription factor snail is a repressor of E-cadherin gene expression in epithelial tumour cells. Nat Cell Biol 2: 84-89, 2000.
42. Yokoyama K, Kamata N, Hayashi E, et al: Reverse correlation of E-cadherin and snail expression in oral squamous cell carcinoma cells in vitro. Oral Oncol 37: 65-71, 2001.

43. Blanco MJ, Moreno-Bueno G, Sarrio D, et al: Correlation of Snail expression with histological grade and lymph node status in breast carcinomas. Oncogene 21: 3241-3246, 2002.

44. Sugimachi K, Tanaka S, Kameyama T, et al: Transcription factor snail and progression of human hepatocellular carcinoma. Clin Cancer Res 9: 2657-2664, 2003.

45. Boire A, Covic L, Agarwal A, Jacques S, Sherifi S and Kuliopulos A: PAR1 is a matrix metalloprotease-1 receptor that promotes invasion and tumorigenesis of breast cancer cells. Cell 120: 303-313, 2005

46. Rømer J, Bugge TH, Pyke C, et al: Plasminogen and wound healing. Nat Med 2: 725, 1996. 